\title{
Prehabilitation in geriatric oncology
}

\author{
Daniel Santa Mina and Shabbir M.H. Alibhai
}

\author{
Version \\ Accepted \\ Citation Santa Mina, D., Alibhai, M.H. Shabbir. \\ (published version) Prehabilitation in geriatric oncology. 2020. Journal of Geriatric \\ Oncology. 11(4) pp731-734. https://doi.org/10.1016/j.jgo.2019.05.017
}

\section{Copyright / License}

CC BY-NC-ND

Publisher's Statement

How to cite TSpace items

Always cite the published version, so the author(s) will receive recognition through services that track citation counts, e.g. Scopus. If you need to cite the page number of the author manuscript from TSpace because you cannot access the published version, then cite the TSpace version in addition to the published version using the permanent URI (handle) found on the record page.

This article was made openly accessible by $U$ of $T$ Faculty. Please tell us how this access benefits you. Your story matters. 
Title: Prehabilitation in Geriatric Oncology

Authors: Daniel Santa Mina ${ }^{1-3}$ and Shabbir M.H. Alibhai2,3

\author{
Affiliations: \\ ${ }^{1}$ Faculty of Kinesiology and Physical Education, University of Toronto, Toronto, Ontario, Canada \\ ${ }^{2}$ Faculty of Medicine, University of Toronto, Toronto, Ontario, Canada \\ ${ }^{3}$ Department of Supportive Care, Princess Margaret Cancer Centre, Toronto, Ontario, Canada
}

Corresponding author:

Dr. Daniel Santa Mina

Faculty of Kinesiology and Physical Education

The University of Toronto

55 Harbord St., Toronto, Ontario, M5S 2W6

416-978-1696

daniel.santamina@utoronto.ca

Word Count: 1599

Tables: 1

References: 22 Maximum 
With advancing age, older adults with cancer often face reductions in functional capacity and comorbid conditions become more prevalent. An overall state of deconditioning can be further exacerbated by a combination of sedentary behaviour and malnutrition that independently contribute to cachexia, morbidity and mortality. ${ }^{1-3}$ Collectively, these changes can induce a state of frailty that undermines the tolerability and safety of cancer treatments. For example, among older adults with cancer undergoing surgery, those who are classified as frail by common multidimensional instruments (e.g., Comprehensive Geriatric Assessment) have a higher risk of surgical complications, longer hospital stays, higher readmission rates, and higher mortality. ${ }^{4,5}$ Given that frailty indices typically capture functional, psychosocial, and/or nutritional assessments, interventions that target deficits in these health domains including exercise, stress reduction, and dietary optimization, respectively, may be particularly valuable for reducing the risk of potential treatment-related adverse effects. In fact, the evidence supporting the role of pretreatment conditioning via one or more targeted health behaviours, referred to as prehabilitation, has rapidly grown in recent years, giving rise to questions about its potential role in models of care. In this commentary, we provide a synopsis of prehabilitation for the older adult with cancer, including a brief review of its origins in geriatric and clinical care, current applications, and a pragmatic implementation model for consideration.

Contemporary prehabilitation research was initiated with efforts to reduce the risk of disability and institutionalization in older adults. Early evidence in this setting was provided by Gill and colleagues' who conducted a randomized controlled trial (RCT) of 188 community-living, frail participants over age 75 and demonstrated less disability with home-based prehabilitation that included muscular strength, mobility, and balance exercises ${ }^{6}$. Compared to controls, intervention participants had $15 \%, 45 \%$, and $37 \%$ less disability development at three, seven, and twelve months, respectively. The concept of prehabilitation was most prominently introduced to the surgical setting by Carli and Zavorsky (2005), who described it as "[a programme] to enhance functional exercise capacity in elderly patients with the intent to minimize the postoperative morbidity and accelerate postsurgical recovery". ${ }^{7}$ Over the last decade, research on prehabilitation in oncology has flourished, with tailored with investigations of its effects in a variety of tumour sites and a cancer-specific definition. ${ }^{8}$ Specifically, multimodal prehabilitation is 
recommended to simultaneously optimize multiple domains of health in preparation for treatment in an effort to maximize peri- and post-treatment benefits. ${ }^{9}$ Furthermore, while prehabilitation has been heavily focused on the surgical patient, emerging data suggest that prehabilitation may also be appropriate prior to chemotherapy or stem cell transplantation, but it is relatively unexplored relative to radiation and targeted therapies.

Few studies have specifically focused on examining prehabilitation in the older adult with cancer. Recent systematic reviews on prehabilitation in the older adult with colorectal cancer undergoing surgery suggest benefits to functional capacity, no effect on postoperative length of stay or hospital readmission rates, and generally low in study quality. ${ }^{10,11}$ We recently reported a feasibility RCT for older men comparing kinesiologist-prescribed, home-based prehabilitation, including moderate intensity aerobic and resistance training plus pelvic floor muscle exercises, versus pelvic floor muscle exercise alone (control condition) in 86 men undergoing radical prostatectomy for prostate cancer. ${ }^{12}$ Compared to control participants, the prehabilitation group reported reduced anxiety prior to surgery by 1.5 points $( \pm 0.7, p=0.035$, anxiety subscale) on the Hospital Anxiety and Depression Scale. At four weeks after surgery, prehabilitated participants recovered to their baseline aerobic functional capacity (as measured by the six-minute walk test) whereas controls exhibited a functional deficit of twice the minimal clinically important difference. In another RCT of prehabilitation, Barberan-Garcia et al ${ }^{13}$ recruited 144 'high-risk' major abdominal surgical candidates, where risk status was partly defined by an age of greater than 70 years. The prehabilitation intervention, prescribed by a physiotherapist, chiefly consisted of daily physical activity promotion (increased volume and intensity of walking per day) and facility-based, supervised high-intensity interval training (HIIT). Prehabilitation resulted in improved aerobic capacity up to the time of surgery and a 51\% decrease in the risk of postoperative complications compared to the control group.

The variability in benefit for prehabilitation in older adults with cancer in the current literature has raised questions about the applicability of generalized intervention design models that fail to account for the often-nuanced needs, capacity, and interests of the older adult. Bruns et al recently conducted a needs assessment to advise prehabilitation intervention development in older adults with cancer, finding preferences for: i) home-based exercise; ii) the prioritization of 
safety and feasibility (i.e., minimal additional exercise equipment or resources required); iii) incentives to participate (rather than a focus on the consequences of non-participation); and iv) the use of technology that "activates the user" to initiate health behaviours. ${ }^{14}$ Their resultant innovation called Fit4SurgeryTV, consists of a touchscreen display embedded into a nostalgic wooden frame that acted as an intervention 'companion' to 'trigger' users to initiate and record prescribed exercise and dietary behaviours prior to surgery. Digital awards displayed were accumulated by participants if they adhered to their exercises and/or meal plans and could be exchanged for passes to the local zoo. In their pilot assessment of fourteen frail colorectal cancer surgery patients aged 70 years or older, most participants reported usability of the device, and prescribed exercises and recipes were completed $86 \%$ and $71 \%$ of the time, respectively. Approximately two thirds of participants reported physical improvement and $86 \%$ felt the zoo passes provided additional motivation. Although intriguing, these results are preliminary given methodological limitations.

Despite limitations in the current evidence base, many clinicians' support prehabilitation in principle. In a recent Delphi study, $80 \%$ or more of colorectal surgeons agreed exercise could be used to improve aerobic performance in patients over the age of 60 awaiting surgery (including explicit agreement for those over 80 years) and a general support for preoperative exercise in respondents' surgical practice. ${ }^{15}$ Similar to clinicians' impression of the value of exercise prior to surgery, a survey conducted by the International Society of Geriatric Oncology found that 56\% of surgical oncologists that assessed nutritional status prior to surgery would consider nutritional prehabilitation. To our knowledge, no studies have asked clinicians about their perceived value or likelihood to prescribe psychological interventions prior to treatment in cancer survivors, likely because it is the most understudied prehabilitation modality. Levett and Grimmett recently summarized the evidence for preoperative psychological interventions in oncology and given the relative scarcity of literature, interpretations of its benefits (within unimodal and multimodal interventions) are cautious and currently limited to only some areas of psychological health (such as depression) and self-reported physical function. ${ }^{16}$ Psychological prehabilitation is positioned to become an established core component of multimodal interventions given the strengthening 
evidence describing the relationship between preoperative mood, attitude, and personality traits and treatment outcomes. ${ }^{16}$

Presently, efforts to optimize health with exercise prior to surgery with prehabilitation in older adults with cancer have been focused on abdominal surgeries, and primarily in people with colorectal cancer. While the data are premature to offer specific guidelines for surgical prehabilitation across treatments (surgery or otherwise), and specifically in the older adult with cancer, it is likely appropriate to promote attaining the World Health Organization targets for physical activity volume (150 minutes of moderate to vigorous aerobic exercise plus two resistance training on two days per week) and apply appropriate adaptations for disease-related and comorbid conditions. Guidelines for nutrition optimization prior to treatment have not been specifically developed for geriatric oncology; however, the American Society for Enhanced Recovery and Perioperative Quality provides a comprehensive set of recommendations for general preoperative nutrition to reduce peri- and postoperative surgical risk that are reasonable strategies to follow until more population-specific data are available. ${ }^{17}$ Research to draw direction for the development of psychological prehabilitation is nascent, particularly with reference to older adults with cancer. Nevertheless, a recent systematic review in people with cancer found that comprehensive, preoperative stress-management may be more beneficial than preoperative psychotherapy, or audio recordings of guided imagery or relaxation alone. ${ }^{18}$

Given evidence that nearly half of all older adult patients awaiting surgery will experience significant functional decline, ${ }^{19}$ it is arguably imperative that a comprehensive, geriatric-focused screen or assessment be implemented to ensure appropriate exercise, nutrition, and psychological prehabilitative care. Early identification of various markers of frailty is consistent with proposed prehabilitation programme models that target the most appropriate candidates towards a costeffective allocation of resources. ${ }^{20,21}$ Furthermore, given the potential risks associated with exercise in the older adults, it is advised that screening and assessment should inform, and if appropriate, conclude with medical clearance for exercise with specific movement contraindications or restrictions to ensure safe participation. Prehabilitation programmatic design considerations with specific reference to the geriatric population are presented in Table 1. 
In spite of current evidence limitations, prehabilitation's potential to reduce treatment toxicity and improve a number of patient-relevant outcomes in older adults with cancer continues

to draw clinical and research attention. The early data support the feasibility and tolerability of prehabilitation in geriatric oncology with potential benefits across a variety of functional and quality of life domains. Also, recent reports highlight clinician support for prehabilitation for older cancer survivors. Calls for the integration of prehabilitation services into standard of care are increasing; however, the most important barrier to wide acceptance and application of prehabilitation is an absence of definitive evidence supporting of its effectiveness. While this evidence gap in evidence is most pronounced in the older and frailer patient, as well as among non-surgical settings, the positive evidence trend and enthusiastic research engagement across health disciplines suggests that decisions about its inclusion into care models for the older cancer survivor are likely a question of 'when' and 'how best' rather than 'if'.

\section{Conflict of Interest / Disclosures:}

The Authors have no conflicts to disclose.

\section{Authors Contributions:}

Conception and Design: DSM and SMHA

Data Collection: N/A

Analysis and Interpretation of Data: N/A

Manuscript Writing: DSM and SMHA

Approval of Final Article. DSM and SMHA

All authors have approved the final article should be true and included in the disclosure.

\section{Acknowledgments:}

We would like to acknowledge the expert insights on nutrition and psychology provided by Chelsia Gillis, RD, MSc and Dr. Andrew G. Matthew, PhD, C.Pysch., respectively. 
1. Arends J, Baracos V, Bertz H, Bozzetti F, Calder PC, Deutz NEP, et al. ESPEN expert group recommendations for action against cancer-related malnutrition. Clin Nutr.

2017;36(5):1187-96. https://doi.org/10.1016/j.clnu.2017.06.017

2. Ryan AM, Power DG, Daly L, Cushen SJ, Ní Bhuachalla E, Prado CM. Cancer-associated malnutrition, cachexia and sarcopenia: The skeleton in the hospital closet 40 years later. Proceedings of the Nutrition Society. 2016;75(2):199-211.

https://doi.org/10.1017/S002966511500419X

3. Kerr J, Anderson C, Lippman SM. Physical activity, sedentary behaviour, diet, and cancer: an update and emerging new evidence. The Lancet Oncology. 2017;18(8):e457-71.

4. Lin HS, Watts JN, Peel NM, Hubbard RE. Frailty and post-operative outcomes in older surgical patients: A systematic review. BMC Geriatrics. 2016;16(1):157. https://doi.org/10.1186/s12877-016-0329-8

5. Feng MA, McMillan DT, Crowell K, Muss H, Nielsen ME, Smith AB. Geriatric assessment in surgical oncology: a systematic review. J Surg Res. 2015;193(1):265-72. https://doi.org/10.1016/j.jss.2014.07.004

6. Gill TM, Baker DI, Gottschalk M, Peduzzi PN, Allore H, Byers A. A program to prevent functional decline in physically frail, elderly persons who live at home. N Engl J Med. 2002;347(14):1068-74. https://doi.org/10.1056/NEJMoa020423

7. Carli F, Zavorsky GS. Optimizing functional exercise capacity in the elderly surgical population. Curr Opin Clin Nutr Metab Care. 2005;8(1):23-32.

8. Silver JK, Baima J, Mayer RS. Impairment-driven cancer rehabilitation: an essential component of quality care and survivorship. CA Cancer J Clin. 2013;63(5):295-317. https://doi.org/10.3322/caac.21186.

9. Carli F, Silver JK, Feldman LS, McKee A, Gilman S, Gillis C, et al. Surgical Prehabilitation in Patients with Cancer. Phys Med Rehabil Clin N Am. 2017;28(1):49-64.

https://doi.org/10.1016/j.pmr.2016.09.002

10. Looijaard SMLM, Slee-Valentijn MS, Otten RHJ, Maier AB. Physical and Nutritional Prehabilitation in Older Patients With Colorectal Carcinoma: A Systematic Review. J Geriatr Phys Ther. 2018;41(4):236-44. https://doi.org/10.1519/JPT.0000000000000125 
11. Bruns ERJ, van den Heuvel B, Buskens CJ, van Duijvendijk P, Festen S, Wassenaar EB, et al. The effects of physical prehabilitation in elderly patients undergoing colorectal surgery: a systematic review. Colorectal Disease. 2016;18(8):0267-77.

https://doi.org/10.1111/codi.13429.

12. Santa Mina D, Hilton WJ, Matthew AG, Awasthi R, Bousquet-Dion G, Alibhai SMH, et al. Prehabilitation for radical prostatectomy: A multicentre randomized controlled trial. Surg Oncol. 2018;27(2):289-98. https://doi.org/10.1016/j.suronc.2018.05.010

13. Barberan-Garcia A, Ubré M, Roca J, Lacy AM, Burgos F, Risco R, et al. Personalised Prehabilitation in High-risk Patients Undergoing Elective Major Abdominal Surgery. Ann Surg.;2018;267(1):50-6. https://doi.org/10.1097/SLA.0000000000002293

14. Bruns ERJ, Argillander TE, Schuijt HJ, van Duijvendijk P, van der Zaag ES, Wassenaar EB, et al. Fit4SurgeryTV at-home prehabilitation for frail elderly planned for colorectal cancer surgery: a pilot study. Am J Phys Med Rehabil. 2018. [Epub ahead of print]. https://doi.org/10.1097/PHM.0000000000001108

15. Boereboom CL, Williams JP, Leighton P, Lund JN. Forming a consensus opinion on exercise prehabilitation in elderly colorectal cancer patients: a Delphi study. Tech Coloproctol. 2015;19(6):347-54. https://doi.org/10.1007/s10151-015-1317-2

16. Levett DZH, Grimmett C. Psychological factors, prehabilitation and surgical outcomes: evidence and future directions. Anaesthesia. 2019;74:36-42. https://10.1111/anae.14507

17. Wischmeyer PE, Carli F, Evans DC, Guilbert S, Kozar R, Pryor A, et al. American Society for Enhanced Recovery and Perioperative Quality Initiative Joint Consensus Statement on Nutrition Screening and Therapy Within a Surgical Enhanced Recovery Pathway. Anesth Analg. 2018;126(6): 1883-95. https://doi.org/10.1213/ANE.0000000000002743

18. Tsimopoulou I, Pasquali S, Howard R, Desai A, Gourevitch D, Tolosa I, et al. Psychological Prehabilitation Before Cancer Surgery: A Systematic Review. Ann Surg Oncol. 2015;22(13):4117-23. https://doi.org/10.1245/s10434-015-4550-z.

19. Palleschi L, Fimognari FL, Pierantozzi A, Salani B, Marsilii A, Zuccaro SM, et al. Acute functional decline before hospitalization in older patients. Geriatr Gerontol Int. 2014;14(4):769-77. https://doi.org/10.1111/ggi.12160 
20. Tew GA, Ayyash R, Durrand J, Danjoux GR. Clinical guideline and recommendations on preoperative exercise training in patients awaiting major non-cardiac surgery. Anaesthesia. 2018;73(6):750-68. https://doi.org/10.1111/anae.14177

21. Minnella EM, Carli F. Prehabilitation and functional recovery for colorectal cancer patients. Eur J Surg Oncol. 2018;44(7):919-26. https://doi.org/10.1016/j.ejso.2018.04.016

22. Carli, F, \& Scheede-Bergdahl, C. Prehabilitation to enhance perioperative care. Anesthesiology clinics. 2015;33(1)17-33.

https://doi.org/10.1016/j.anclin.2014.11.002 